\title{
Caracterização da produção científica acerca das lesões por fricção: revisão
}

\section{integrativa}

\author{
Characterization of scientific production about friction injuries: integrative review \\ Caracterización de la producción científica sobre lesiones por fricción: una revisión integradora
}

Recebido: 21/08/2021 | Revisado: 28/08/2021 | Aceito: 29/08/2021 | Publicado: 02/09/2021

Ravana Amália Ribeiro Barreto

ORCID: https://orcid.org/0000-0003-2677-8198 Universidade Federal do Rio Grande do Norte, Brasil

E-mail: ravanabarreto@gmail.com

Albenize de Azevedo Soares

ORCID: https://orcid.org/0000-0002-3008-0043 Nome da instituição onde atua, País

E-mail: nize.azevedo@hotmail.com

Ana Beatriz Dantas do Nascimento

ORCID: https://orcid.org/0000-0002-6837-096X Universidade Federal do Rio Grande do Norte, Brasil E-mail ana030813@hotmail.com

Ivani Iasmim de Araújo

ORCID: https://orcid.org/0000-0002-2452-2263 Universidade Federal do Rio Grande do Norte, Brasil E-mail: yasminaraujo88@gmail.com

Jayara Mikarla de Lira

ORCID: https://orcid.org/0000-0002-1707-0983 Universidade Federal do Rio Grande do Norte, Brasil E-mail: jayara-mikarla@hotmail.com

Ilisdayne Thallita Soares da Silva

ORCID: https://orcid.org/0000-0003-2421-8090 Universidade Federal do Rio Grande do Norte, Brasil E-mail: ilisdayne@gmail.com

\begin{abstract}
Resumo
Objetivo: identificar na literatura as produções científicas sobre lesões do tipo Skin Tears. Metodologia: revisão integrativa, cuja busca ocorreu entre abril e junho de 2020, nas bases de dados LILACS, PubMed, SCOPUS, SciELO, Web of Science e Cochrane para responder à questão norteadora: "Quais as características da produção científica sobre lesões do tipo Skin Tears?". Foram incluídos artigos de pesquisa originais que atendiam ao objetivo da pesquisa. Excluíram-se estudos da literatura cinzenta (relatos de caso, reflexão, recomendações), artigos de revisão e estudos que focavam outras temáticas. Resultados: as buscas nas bases de dados pesquisadas capturaram 371 referências. Após as exclusões, selecionaram-se 13 estudos para serem lidos na íntegra, onde destes, 07 artigos foram selecionados para compor esta revisão, agrupados empiricamente, conforme suas similaridades, em três categorias: "Prevalência e fatores de risco para as Skin Tears", "Importância da prevenção, e manejo das Skin Tears" e "Fragilidades de protocolos institucionais que padronizem o cuidado das Skin Tears". Conclusão: a abordagem teórica sobre as lesões por fricção é primordial para prestar uma assistência efetiva, visando minimizar o desconforto e as complicações em pacientes acometidos por estas. Entretanto, constatou-se escassez de estudos que possibilitem fundamentar uma discussão de qualidade, dado que é imprescindível a promoção de uma assistência integral que garanta melhor qualidade de vida.

Palavras-chave: Pele; Atenção à saúde; Fricção; Ferimento.
\end{abstract}

\begin{abstract}
Objective: to identify scientific productions on Skin Tears injuries in the literature. Methodology: integrative review, searched between April and June 2020, in LILACS, PubMed, SCOPUS, SciELO, Web of Science and Cochrane databases to answer the guiding question: "What are the characteristics of scientific production on Skin-type injuries Tears?". Original research articles that met the research objective were included. Gray literature studies (case reports, reflection, recommendations), review articles and studies that focused on other themes were excluded. Results: searches in the surveyed databases captured 371 references. After the exclusions, 13 studies were selected to be read in full, of which, 07 articles were selected to compose this review, empirically grouped, according to their similarities, into three categories: "Prevalence and risk factors for Skin Tears", "Importance of prevention and management of Skin Tears" and "Weaknesses of institutional protocols that standardize the care of Skin Tears". Conclusion: the theoretical approach to friction injuries is essential to provide effective care, aiming to minimize discomfort and complications in patients
\end{abstract}


affected by them. However, there was a scarcity of studies that could support a discussion of quality, as it is essential to promote comprehensive care that guarantees a better quality of life.

Keywords: Skin; Health care; Friction; Injury.

\begin{abstract}
Resumen
Objetivo: identificar producciones científicas sobre lesiones cutáneas lagrimales en la literatura. Metodología: revisión integradora, investigada entre abril y junio de 2020, en las bases de datos LILACS, PubMed, SCOPUS, SciELO, Web of Science y Cochrane para responder a la pregunta orientadora: “¿Cuáles son las características de la producción científica sobre Lesiones? ¿Te gustan las lágrimas en la piel?”. Se incluyeron artículos de investigación originales que cumplieron con el objetivo de la investigación. Se excluyeron los estudios de literatura gris (informes de casos, reflexión, recomendaciones), artículos de revisión y estudios centrados en otros temas. Resultados: las búsquedas en las bases de datos encuestadas capturaron 371 referencias. Tras las exclusiones, se seleccionaron 13 estudios para lectura completa, de los cuales se seleccionaron 07 artículos para componer esta revisión, agrupados empíricamente, según sus similitudes, en tres categorías: "Prevalencia y factores de riesgo de desgarro cutáneo", "Importancia de la Prevención y Manejo de Lesiones de la Piel "y“ Debilidades de los protocolos institucionales que estandarizan la atención de las Lesiones de la Piel ". Conclusión: el abordaje teórico de las lesiones por fricción es fundamental para brindar una atención eficaz, con el objetivo de minimizar las molestias y las complicaciones - falta de estudios que sustenten la discusión sobre la calidad, ya que es fundamental promover una atención integral que garantice una mejor calidad de vida.
\end{abstract}

Palabras clave: Piel; Cuidados de la salud; Fricción; Perjudicar.

\title{
1. Introdução
}

As Lesões por Fricção (LFs) ou do tipo Skin Tears são feridas dolorosas resultantes de traumas cutâneos que afetam pessoas com a pele frágil, podendo ser causadas por fricção, cisalhamento ou contusão, cuja pressão acarreta a separação das camadas da pele (epiderme e derme) e, consequentemente, a formação de um retalho. Essa separação pode ser de espessura parcial, a qual acomete a epiderme ou derme, ou de espessura total com separação da hipoderme até a fáscia. As LFs são classificadas em categorias $1 \mathrm{a}, 1 \mathrm{~b}, 2 \mathrm{a}, 2 \mathrm{~b}$ e 3 de acordo com a avaliação clínica de pele. Seu desenvolvimento varia de lesões sem alterações na coloração da pele, com presença de retalho susceptível de realinhamentoe até a perda completa do retalho (Tristão, Girondi, Hammerschmidt, Soares, Martins \& Lima, 2018).

As LFs são mais prevalentes do que a lesão por pressão (LPP), especialmente no Brasil. Nos Estados Unidos, ocorrem mais de 1,5 milhão de lesões do tipo Skin Tears a cada ano em idosos internados. Estima-se que, até 2030, o número de indivíduos de alto risco para essas lesões seja de 8,1 milhões de pessoas, somente nesse país (Gomes, Souza, Rocha, Kuriyama \& Silvino, 2016).

Os fatores de risco para desencadear a LFs são: uso prolongado de medicamentos esteróides, nutrição deficiente, mobilidade, cognição prejudicada e equimose. Assim, caracteriza-se como grupo de risco os idosos, pacientes em cuidados críticos, com doenças crônicas e pacientes que necessitam de auxílio para as atividades de vida diária. As LFs podem acontecer em qualquer parte do corpo, no entanto a maior ocorrência é nas extremidades dos membros superiores, inferiores, costas e nádegas (Soh Z, et al., 2019; Lopes et al., 2011).

$\mathrm{Na}$ prática assistencial da equipe de enfermagem é fundamental que os profissionais tenham conhecimento acerca do tema, pois estas lesões são comumente dolorosas e podem desencadear complicações, como infecções e sangramentos, as quais são prevenidas com algumas medidas, como a implementação de escalas de avaliação de risco validadas, promoção da higiene corporal adequada, manejo de mobilidade e manutenção da umidade da pele do paciente (Gomes et al., 2016; Aranha et al., 2020).

Umas das grandes problemáticas que envolve o tema é o pouco conhecimento que se tem a respeito e a falta de disseminação de informações corretas com base científica. Outra grande questão é a incidência da LFs, que acomete mais a população idosa, a qual, na realidade brasileira, mantém a tendência de crescimento e ultrapassa os 30 milhões de pessoas (IBGE, n.d.). Consequentemente a estes fatores, o manejo a esses pacientes apresenta desafios para os profissionais de saúde. Para isso, 
a prática de enfermagem deve estar baseada na informação de caráter científico a fim de proporcionar avaliação, mecanismos de prevenção e de tratamento apropiados para esse tipo de lesão (Gomes, Souza, Rocha, Kuriyama \& Silvino, 2016).

A partir disso, considera-se que o levantamento da literatura científica sobre essa lesão possibilita subsídios para a criação de estratégias de promoção, prevenção e tratamento em saúde, na qual a enfermagem está inserida. Nessa perspectiva, o estudo objetiva caracterizar a produção científica sobre LFs ou do tipo Skin Tears visando identificar as lacunas a respeito desse conteúdo.

\section{Metodologia}

Trata-se de uma pesquisa que utilizou como método a revisão de literatura do tipo integrativa, a qual permite realizar a busca, a análise e a seleção de produções científicas, possibilitando a síntese do conhecimento sobre determinado assunto e expondo a necessidade de novos estudos (Soares et al., 2014; Faria, 2010).

Para construção da pesquisa, seguiram os seguintes estágios: elaboração da questão de pesquisa, busca na literatura, coleta de dados, análise crítica dos estudos incluídos, discussão dos resultados e apresentação da revisão (Faria, 2010)

Para direcionamento desta pesquisa, foi elaborada a seguinte questão norteadora: "Quais as características da produção científica sobre lesões do tipo Skin Tears?"

O levantamento das publicações ocorreu no período de maio e junho de 2020 nas referidas bases de dados: Literatura Latino-Americana e do Caribe em Ciências da Saúde (LILACS), PubMed, Scopus, Web of Science, The Scientific Electronic Library Online (SciELO), Cochrane e Cinahl, não sendo estabelecidos limites quanto ao ano de publicação.

Para o refinamento dos artigos estabeleceram-se como critérios de inclusão: artigos publicados em português, espanhol e inglês e que atendessem ao objetivo da pesquisa. Foram excluídos documentos em formato de editorial, artigo de reflexão, revisão de literatura, trabalho de conclusão de curso, teses, dissertações e artigos repetidos.

Para busca foram selecionados os descritores controlados do vocabulário Medical Subject Heading (MeSH), na língua inglesa: Skin; Wounds and Injuries; Friction. O cruzamento desses descritores ocorreu por meio do operador booleano AND.

Com o objetivo de assegurar maior confiabilidade a esse estudo, foi utilizado o documento Preferred Reporting Items for Systematic Reviews and Meta-Analysis (PRISMA). O PRISMA consiste em um checklist de 27 pontos imprescindíveis para guiar o estudo de forma clara e detalhada que pode ainda, ser visualizada em um fluxograma com todas as fases de desenvolvimento do estudo, também acompanhado de um documento explicativo e exemplificado (Fuchs \& Paim, 2010).

Apesar do PRISMA ter sido desenvolvido e ser recomendado para revisões sistemáticas e metanálises a fim de aumentar sua qualidade (Fuchs \& Paim, 2010), foi possível sua utilização nessa revisão integrativa por meio da exclusão dos itens do checklist que são específicos para revisões sistemáticas.

O nível de evidência atribuído aos artigos baseou-se na seguinte classificação: nível I - evidências resultantes de revisões sistemáticas ou meta-análise de ensaios clínicos; nível II - evidências oriundas de no mínimo um ensaio clínico randomizado bem delineado; nível III - ensaios clínicos sem randomização; nível IV - estudos de coorte e caso-controle com um bom delineamento; nível V - revisão sistemática de estudos descritivos e qualitativos; nível VI - evidências provenientes de um único estudo descritivo e qualitativo; nível VII - evidências baseadas em opinião de autoridades ou comitês de especialistas (Melnyk, Fineout-Overholt, Stillwell, \& Williamson, 2010). 
Tabela 1 - Número de artigos obtidos nas bases de dados no período de maio a junho de 2020.

\begin{tabular}{ccccc}
\hline $\begin{array}{c}\text { Bases de } \\
\text { Dados }\end{array}$ & $\begin{array}{c}\text { Artigos } \\
\text { Encontrados }\end{array}$ & $\begin{array}{c}\text { Artigos } \\
\text { Excluídos }\end{array}$ & $\begin{array}{c}\text { Artigos } \\
\text { Selecionados }\end{array}$ & $\begin{array}{c}\text { Artigos } \\
\text { analisados }\end{array}$ \\
LILACS & 9 & 8 & 1 & 1 \\
PubMed & 127 & 125 & 2 & 2 \\
SCOPUS SciELO & 121 & 119 & 2 & 1 \\
Webof Science & 4 & 2 & 2 & 2 \\
Cochrane & 44 & 43 & 1 & 0 \\
Cinahl & & 13 & 2 & 0 \\
& 15 & 48 & 3 & 1 \\
\hline
\end{tabular}

Fonte: Autores.

O Fluxograma 1 apresenta o processo de elaboração da revisão a partir do PRISMA.

Fluxograma 1 - Processo de seleção, elegibilidade e inclusão dos artigos na revisão integrativa elaborado a partir da recomendação PRISMA. Santa Cruz, Rio Grande do Norte, Brasil, 2020.
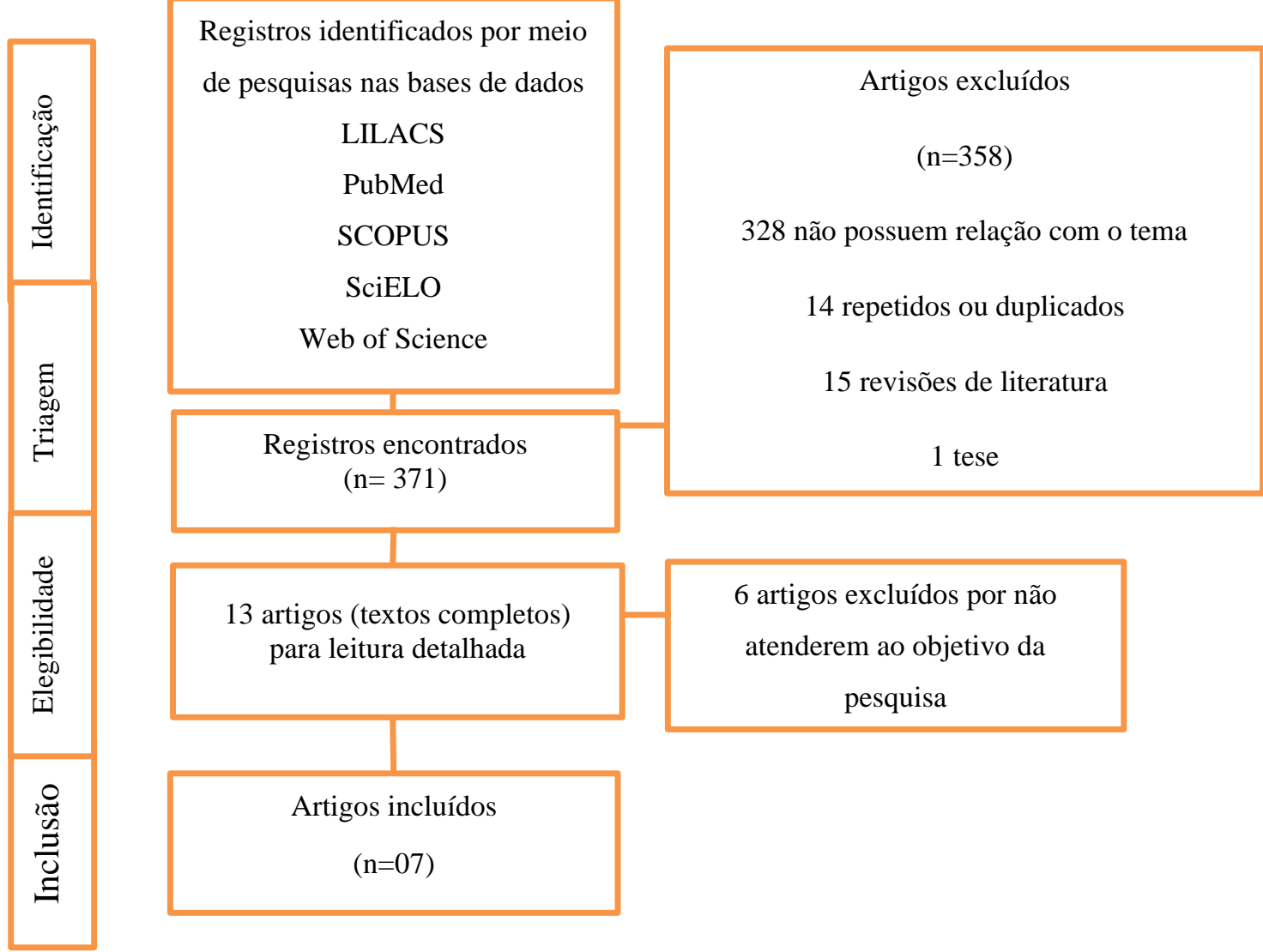

Fonte: Autores (2021).

\section{Resultados}

Dos 7 artigos elegidos para a análise, 4 foram publicados em periódicos de enfermagem e os demais em periódicos de medicina e biomedicina. Destes, 2 foram publicados na revista Cogitare Enfermagem, seguido da International Journal of Nursing Practice (1), International Journal of Evidence-Based Healthcare (1), Pilotand Feasibility Studies (1), Revista da Escola de Enfermagem da USP (1) e Texto \& Contexto - Enfermagem (1). 
Os artigos examinados concentram-se no período de 2011 a 2020. A população dos estudos consistiu em pacientes idosos (6) e pacientes oncológicos (1).

No que se refere ao local de estudo, dois foram executados em Unidades de Terapia Intensiva, seguido de Unidades de Cuidados Agudos e de Reabilitação, Hospital de Urgência, Instituição de Longa Permanência, Unidades Básicas de Saúde e Lares de idosos. No tocante ao país de publicação, quatro foram realizados no Brasil, um na Austrália, um no Reino Unido e um em Cingapura. Quanto ao idioma dos estudos, dois são na língua portuguesa e os demais na língua inglesa.

Conforme a caracterização metodológica da pesquisa dos artigos analisados, detectou-se que a maioria utilizou a abordagem transversal (6) e ensaio clínico (1). Quanto ao tipo de delineamento, três foram de cunho descritivo, dois analítico, um exploratório e um experimental. Dessa forma, em relação à força de evidência, observou-se que seis artigos apresentam nível de evidência VI e um artigo nível de evidência II.

A Tabela 2 exibe a síntese das informações dos artigos científicos incluídos na revisão integrativa sobre LFs ou do tipo Skin Tears.

Tabela 2 - Produção científica sobre LFs ou do tipo Skin Tears. Santa Cruz, Rio grande do Norte, Brasil, 2020.

\begin{tabular}{|c|c|c|c|c|c|c|c|}
\hline Título & $\begin{array}{l}\text { Autor/Base de } \\
\text { dados }\end{array}$ & Periódico/Ano & Objetivo & $\begin{array}{l}\text { Local } \\
\text { estudo }\end{array}$ & $\begin{array}{l}\text { Tipo } \\
\text { estudo/nível } \\
\text { evidência }\end{array}$ & $\begin{array}{l}\text { de } \\
\text { de }\end{array}$ & $\begin{array}{l}\text { Síntese } \\
\text { resultados }\end{array}$ \\
\hline $\begin{array}{l}\text { Skin tear prevention } \\
\text { and management } \\
\text { among patients in the } \\
\text { acute aged care and } \\
\text { rehabilitation units in } \\
\text { the Australian Capital } \\
\text { Territory: a best } \\
\text { practice } \\
\text { implementation } \\
\text { project }\end{array}$ & $\begin{array}{l}\text { Lopez, V.; Dunk, } \\
\text { A. M.; Cubit, K.; } \\
\text { Parke, J.; Larkin, } \\
\text { D.; Trudinger, M.; } \\
\text { Stuart, } \\
\text { CINAHL }\end{array}$ & $\begin{array}{l}\text { International } \\
\text { Journal of } \\
\text { Evidence-Based } \\
\text { Healthcare/ } \\
2011\end{array}$ & $\begin{array}{l}\text { Garantir a prática } \\
\text { de avaliação, de } \\
\text { prevenção e de } \\
\text { gestão da LFs entre } \\
\text { pacientes em } \\
\text { unidades de } \\
\text { cuidados intensivos } \\
\text { e de reabilitação. }\end{array}$ & $\begin{array}{l}\text { Hospitais } \\
\text { públicos da } \\
\text { Capital } \\
\text { Australiana }\end{array}$ & $\begin{array}{l}\text { Descritivo, } \\
\text { transversal, } \\
\text { abordagem } \\
\text { quantitativa } \\
\text { qualitativa/ VI }\end{array}$ & e & $\begin{array}{l}\text { A implementação de } \\
\text { um projeto de } \\
\text { intervenção auxilia na } \\
\text { prevenção e no } \\
\text { gerenciamento de LF. }\end{array}$ \\
\hline
\end{tabular}

\begin{tabular}{|c|c|c|c|c|c|c|}
\hline $\begin{array}{l}\text { Prevalence of } \\
\text { Friction Injuryand } \\
\text { Associated Factors in } \\
\text { Elderly in Intensive } \\
\text { Therapy }\end{array}$ & 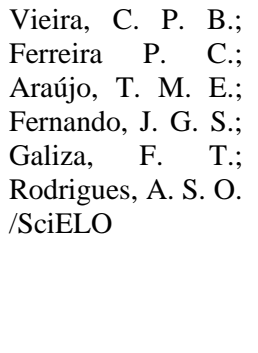 & $\begin{array}{l}\text { Texto\&Contexto } \\
\text {-Enfermagem } \\
/ 2020\end{array}$ & $\begin{array}{l}\text { Analisar } \\
\text { prevalência de } \\
\text { Lesões por fricção e } \\
\text { fatores associados } \\
\text { em } r \text { idosos } \\
\text { internados } \\
\text { Unidade de } \\
\text { Intensiva. }\end{array}$ & $\begin{array}{l}\text { Unidade de } \\
\text { terapia } \\
\text { intensiva de } \\
\text { Hospital } \\
\text { Escola, } \\
\text { Brasil. }\end{array}$ & $\begin{array}{l}\text { Estudo } \\
\text { transversal } \\
\text { analítico/VI }\end{array}$ & $\begin{array}{l}\text { Há uma prevalência } \\
\text { moderada de LF nos } \\
\text { idosos e estes } \\
\text { apresentam em média } \\
\text { mais de uma lesão. } \\
\text { Verificou-se } \\
\text { associação deste tipo } \\
\text { de lesão com a idade, } \\
\text { comorbidades, pele } \\
\text { seca e descamativa }\end{array}$ \\
\hline $\begin{array}{l}\text { Prevalência de } \text { Lesão } \\
\text { por fricção em } \\
\text { pacientes } \\
\text { hospitalizados } \\
\text { câncer }\end{array}$ & $\begin{array}{l}\text { Amaral, A. F. S.; } \\
\text { Pulido, K. C. S.; } \\
\text { Santos, V. L. C. G./ } \\
\text { SCielo }\end{array}$ & $\begin{array}{l}\text { Revista Escola de } \\
\text { Enfermagem da } \\
\text { USP/2012 }\end{array}$ & $\begin{array}{l}\text { Identificar a } \\
\text { prevalência de } \\
\text { Lesões por fricção } \\
\text { (LFs) em pacientes } \\
\text { hospitalizados com } \\
\text { câncer e avaliar os } \\
\text { fatores e } \\
\text { demográficos seu } \\
\text { clínicos associados } \\
\text { ao desenvolvimento. } \\
\text { de }\end{array}$ & $\begin{array}{l}\text { Instituto do } \\
\text { Câncer do } \\
\text { Estado de } \\
\text { São Paulo, } \\
\text { Brasil }\end{array}$ & $\begin{array}{l}\text { Estudo } \\
\text { epidemiológico, } \\
\text { exploratório e } \\
\text { transversal /VI }\end{array}$ & $\begin{array}{l}\text { Houve baixa } \\
\text { prevalência de LFs } \\
\text { entre os pacientes. } \\
\text { Verificou-se que essas } \\
\text { lesões estão associadas } \\
\text { a menor independência } \\
\text { e autonomia para o } \\
\text { autocuidado. }\end{array}$ \\
\hline $\begin{array}{l}\text { Risk of Skin Tears } \\
\text { and its predictors } \\
\text { among hospitalized } \\
\text { older adults in } \\
\text { Singapore }\end{array}$ & $\begin{array}{l}\text { Soh, Z.; Wang, W.; } \\
\text { Png, G. K.; Hassan, } \\
\text { N.; Wu, V. X./ } \\
\text { PubMed }\end{array}$ & $\begin{array}{l}\text { International } \\
\text { journal of nursing } \\
\text { practice / } 2019\end{array}$ & $\begin{array}{lr}\text { Identificar } & \text { as } \\
\text { características } & \text { que } \\
\text { podem } & \text { ser } \\
\text { preditivas } & \text { de } \\
\text { desenvolvimento } & \\
\text { de LF entre idosos } \\
\text { hospitalizados em } \\
\text { Cingapura. }\end{array}$ & $\begin{array}{l}\text { Hospital de } \\
\text { urgência em } \\
\text { Cingapura. }\end{array}$ & $\begin{array}{l}\text { Estudo } \\
\text { transversal } \\
\text { descritivo / VI }\end{array}$ & $\begin{array}{l}\text { Constatou-se que a } \\
\text { idade, baixo peso, } \\
\text { dependência do } \\
\text { cuidador e demência } \\
\text { previram níveis mais } \\
\text { elevados de LF entre } \\
\text { idosos hospitalizados. }\end{array}$ \\
\hline
\end{tabular}




\begin{tabular}{|c|c|c|c|c|c|c|c|}
\hline $\begin{array}{l}\text { Prevalência de lesões } \\
\text { por fricção em idosos } \\
\text { institucionalizados }\end{array}$ & $\begin{array}{l}\text { Vieira, C. P. B.; } \\
\text { Araújo, T. M. E.; } \\
\text { Júnior, F. J. } \\
\text { Rodrigues, A. } \\
\text { O.; Galiza, F. T./ } \\
\text { LILACS }\end{array}$ & $\begin{array}{l}\text { Cogitare } \\
\text { Enfermagem/ } \\
2019\end{array}$ & $\begin{array}{l}\text { Analisar } \\
\text { prevalência de } \\
\text { lesões por fricção e } \\
\text { os fatores de risco } \\
\text { associados } \\
\text { idosos }\end{array}$ & $\begin{array}{l}\text { instituição de } \\
\text { longa } \\
\text { permanência } \\
\text { em Teresina, } \\
\text { Piauí }\end{array}$ & $\begin{array}{l}\text { Estudo } \\
\text { transversal } \\
\text { analítico / VI }\end{array}$ & $\mathrm{e}$ & $\begin{array}{l}\text { Observou-se elevada } \\
\text { prevalência de LFs. A } \\
\text { maioria das lesões } \\
\text { localizada nos } \\
\text { membros inferiores e } \\
\text { de categoria 3. }\end{array}$ \\
\hline
\end{tabular}

\begin{tabular}{|c|c|c|c|c|c|c|}
\hline $\begin{array}{l}\text { Pilot parallel } \\
\text { randomised } \\
\text { controlled trial of } \\
\text { protective socks } \\
\text { against usual care to } \\
\text { reduce Skin Tears in } \\
\text { high risk people: } \\
\text { 'STOPCUTS' }\end{array}$ & $\begin{array}{l}\text { Powell, R. J.; } \\
\text { Hayward, C. J.; } \\
\text { Snelgrove, C. L.; } \\
\text { Polverino, K.; Park, } \\
\text { L.; Chauhan, R.; } \\
\text { Evans, P. H.; } \\
\text { Byford, } \\
\text { Charman C.; Foy, } \\
\text { C. J. H.; Kingsley, } \\
\text { A./ PubMed }\end{array}$ & $\begin{array}{l}\text { Pilot Feasibility } \\
\text { Stud/ } 2017\end{array}$ & $\begin{array}{l}\text { Avaliar a eficácia e } \\
\text { relação custo- } \\
\text { benefício das meias } \\
\text { dermatuff na } \\
\text { proteção dos } \\
\text { membros inferiores } \\
\text { e na prevenção de } \\
\text { lesões por fricção } \\
\text { em idosos }\end{array}$ & $\begin{array}{l}\text { Lares de } \\
\text { idosos em } \\
\text { Devon, } \\
\text { Reino Unido. }\end{array}$ & $\begin{array}{l}\text { Ensaio clínico } \\
\text { randomizado /II }\end{array}$ & $\begin{array}{l}\text { O uso de meias } \\
\text { Dermatuff resultou em } \\
\text { reduções na incidência } \\
\text { de LF, nos custos para } \\
\text { o tratamento, além de } \\
\text { ter sido associada com } \\
\text { melhor qualidade de } \\
\text { vida relacionada a } \\
\text { saúde. }\end{array}$ \\
\hline $\begin{array}{l}\text { Nursing care } \\
\text { practices in primary } \\
\text { health care: Elderly } \\
\text { people's skin care } \\
\text { management }\end{array}$ & $\begin{array}{l}\text { Tristão, F. R.; } \\
\text { Girondi, J. B. R.; } \\
\text { Hammer scmidt, K. } \\
\text { S. A.; Zamprogna, } \\
\text { K. M.; Soares, C. } \\
\text { F.; Evaristo, S. M.; } \\
\text { Vieira, A. S./ } \\
\text { Scopus }\end{array}$ & $\begin{array}{l}\text { Cogitare } \\
\text { Enfermagem } \\
2020\end{array}$ & $\begin{array}{l}\text { Identificar práticas } \\
\text { de cuidado } \\
\text { empregadas pelos } \\
\text { Enfermeiros da } \\
\text { Estratégia Saúde da } \\
\text { Família para } \\
\text { prevenção, } \\
\text { diagnóstico } \\
\text { enfermagem de } \\
\text { tratamento de LFs e } \\
\text { lesão por pressão } \\
\text { em idosos na } \\
\text { comunidade. }\end{array}$ & $\begin{array}{l}\text { Unidades } \\
\text { básicas de } \\
\text { saúde de São } \\
\text { José, Santa } \\
\text { Catarina }\end{array}$ & $\begin{array}{l}\text { Qualitativo } \\
\text { descritivo/VI }\end{array}$ & $\begin{array}{l}\text { Verificou-se a } \\
\text { ausência de protocolos } \\
\text { institucionais para a } \\
\text { condução do cuidado } \\
\text { às LF, sendo comuns } \\
\text { divergências quanto às } \\
\text { práticas de cuidado } \\
\text { exercidas, e aleatória, } \\
\text { quando presente, a } \\
\text { seleção de ferramentas } \\
\text { para o gerenciamento } \\
\text { do risco e manejo de } \\
\text { ambas as lesões. Os } \\
\text { enfermeiros deter } \\
\text { demonstraram } \\
\text { maior conhecimento } \\
\text { sobre as LP e pouca } \\
\text { familiaridade quanto à } \\
\text { LFs. }\end{array}$ \\
\hline
\end{tabular}

Fonte: Autores (2021).

Com base na análise dos artigos, definiu-se a construção de três eixos temáticos a partir dos achados acerca da caracterização da produção científica sobre lesões do tipo Skin Tears, quais sejam: "Prevalência e fatores de risco para as Skin Tears", "Importância da prevenção e manejo das Skin Tears" e "Fragilidades de protocolos institucionais que padronizem o cuidado das Skin Tears".

\section{Discussão}

\section{Prevalência e fatores de risco para as Skin Tears}

As LFs é uma lesão traumática que atinge os extremos de idade, ou seja, grupos que necessitam de dependência para a realização das atividades básicas de vida diária. Alguns estudos retratam que as LFs são mais prevalentes que as LPP e queimaduras, na qual regularmente são más diagnosticadas, sendo comumente confundidas com laceração cutânea, o que implica no tratamento clínico inadequado (Lima et al., 2021). Uma revisão da literatura mostrou que a prevalência se encontra em torno de 3,3\% a 22\%, já em comparação a outros estudos essa prevalência se torna variável e associada diretamente a idade avançada e a diminuição das funções da pele (LeBlanc et al., 2018).

O maior risco de desenvolvimento das Skin Tears é em pacientes idosos em decorrência do processo de envelhecimento, no qual a pele torna-se mais frágil e vulnerável a danos. Alguns fatores de risco intrínsecos para o desenvolvimento das LFs são: perda de colágeno, diminuição da espessura da epiderme/derme e redução da atividade das glândulas sudoríparas. Há outras 
condições clínicas, como diabetes mellitus e hipertensão arterial que são responsáveis pelo agravamento das lesões devido susceptibilidade da diminuição da vascularização, retardando assim a cicatrização ${ }^{(11,12)}$.

Em relação aos fatores extrínsecos, os ambientes domiciliares e hospitalares são incluídos nessa classificação, visto que são os locais de maior ocorrência deste tipo de lesão, pois são os meios mais frequentes de realização das atividades diárias de cuidado, tais como: mobilidade, vestimenta e higiene, sendo necessário a suspensão do uso de adornos, unhas cortadas e lixadas, acolchoar e/ou cobrir áreas e dispositivos potenciais de risco (LeBlanc et al., 2018).

As regiões do corpo mais acometidas são as extremidades dos membros superiores, as nádegas, costas e os membros inferiores. Assim, é imprescindível estar atento a essas localidades para evitar o surgimento, até mesmo a reincidência das lesões (Holmes, Davidson, Thompson \& Kelechi, 2013).

O reconhecimento dos elementos que compõem a construção da prevalência das Skin Tears é de suma importância para a aplicação correta de cuidados. A identificação e avaliação holística da pele, mobilidade e quadro de saúde geral do paciente são peças fundamentais para a construção do planejamento de ações de cuidados que atenuem os danos a pele.

\section{Importância da prevenção e manejo das Skin Tears}

Apesar de não existir um instrumento específico para identificação dos fatores de risco para a ocorrência das LFs, o reconhecimento desses fatores, bem como os cuidados com a pele, com a mobilidade e o estado geral do paciente, se mostram essenciais como mecanismos de prevenção (Torres, Blanes, Galvão \& Ferreira, 2017).

As práticas de cuidados da equipe de enfermagem devem ser voltadas para uma avaliação cautelosa na pele das pessoas com elevado risco de desenvolver LFs, para possibilitar a realização de diagnósticos de enfermagem em tempo oportuno e em seguida criar intervenções com intuito de prevenir essas lesões como também tratá-las de forma apropriada, evitando possíveis complicações decorrentes (Tristão et al., 2020).

Nesse contexto, Lopez et al., (2011), Soh et al., (2019) e Powell et al., (2020) citam diversas medidas visando a prevenção das LFs e as complicações decorrentes destas, como: ofertar um ambiente livre de perigos, avaliar os aspectos nutricionais, examinar diariamente e criteriosamente a pele de pacientes com fatores de risco para LFs, realizar hidratação diária da pele com hidratantes emolientes principalmente nos locais de maior incidência, efetuar o banho com sabonete de $\mathrm{PH}$ balanceado, tratar com maior atenção as áreas com saliência óssea devido alguns movimentos produzirem cisalhamento excessivo ou fricção, o que pode resultar em lacerações na pele, fazer uso de protetores de espumas nas camas e nas cadeiras como apoio e proteção para os membros superiores e inferiores, como exemplo as meias Dermatuff.

Devidamente registradas na Agência Reguladora de Medicamentos e Produtos para a Saúde (MHRA), as meias Dermatuff constituem um dispositivo médico de classe I, com função de promover proteção contra LFs. As meias possuem na seç̧ão de perna tecido com fibras Kevlar e nylon elástico, conferindo elasticidade e fornecendo um tecido externo duro, resistente a cortes e abrasões. Existe em seu interior uma malha loops que viabiliza uma sensação de almofada e resistência a impactos e ionização (Powell et al., 2017).

Em seu estudo piloto randomizado, com população de 90 pessoas em potencial risco de desenvolver LFs, Powell et al., (2017) observou que 8 dos 44 participantes do grupo de intervenção que fizeram uso das meias Dermatuff foram acometidas pela LFs. Em contrapartida, no grupo controle, dos 46 participantes que não utilizou as meias 10 apresentaram LFs, como também estas surgiram em um tempo médio mais curto, com episódios repetidos, lesões maiores e mais graves.

No entanto, algumas dificuldades são relatadas no uso das meias, como sensação de calor, edema do membro, surgimento de bolha, coceira, necessidades de calçar e retirar as meias levando a alguns indivíduos a precisar de ajuda e não considerar usá-las para socializar com roupas elegantes (Powell et al., 2017). 
Na prática clínica verifica-se a necessidade de estabelecer um protocolo institucional para prevenção e tratamento de LFs, visto que o tratamento realizado na unidade de saúde não tem embasamento científico e algumas das práticas utilizadas, como controle de umidade, hidratação e reposicionamento, caracterizam-se como medidas preventivas (TRISTÃO et al., 2020).

Em virtude do déficit de protocolos, há algumas recomendações de Lopez et al., (2011), Amaral et al., (2012) e Tristão et al., (2020), tais como: aproximar as bordas e aplicar hidrogel, alginato de prata, espumas de silicone ou curativo de biocelulose de acordo com a complexidade da lesão e indicar que a direção para remoção dos curativos deve ser o lado oposto a laceração. Não é recomendado o uso de qualquer adesivo ou produto que permaneça no local por vários dias.

Nesse contexto, é função da equipe de enfermagem estabelecer protocolos de cuidados gerais com a pele, fundamentado em evidências científicas, visando promover essa reparação tecidual e, consequentemente, propiciar um bem-estar ao paciente (Holmes et al., 2013).

\section{Fragilidades de protocolos institucionais que padronizem o cuidado das Skin Tears}

Os Procedimentos Operacionais Padrão (POP) consistem em instrumentos gerenciais modernos, que objetivam a padronização das intervenções e dos procedimentos. Sua utilização permite melhorias na qualidade da assistência prestada através da padronização dos cuidados que os profissionais prestam a seus pacientes, garantindo maior satisfação e segurança para o profissional e para o paciente de acordo com os princípios técnico-científicos.

Os estudos analisados nesta revisão constatam a fragilidade de protocolos institucionais que padronizam a avaliação e subsequente intervenção no que se refere às LFs, capazes de tratar ou prevenir complicações inerentes à alteração ou perda da integridade cutânea que as causam. Em conformidade aos achados, Tristão et al., (2020) relata que para a avaliação da LFs os enfermeiros da Estratégia de Saúde da Família, apontam o uso de diversas ferramentas, tais como, o protocolo de prevenção de lesão por pressão do Ministério da Saúde, a escala de Braden, a escala de Norton e a avaliação clínica, sem nenhum uso de protocolo específico.

Existe um protocolo para identificação, prevenção e tratamento das LFs denominado Skin Tear Classification System (STAR) criado por Payne e Martin (1990) e revisado por Carville et al., (2007). No Brasil, Strazieri-Pulido (2010) fez um estudo deste protocolo com propósito de realizar uma adaptação transcultural e validação desse instrumento para a língua portuguesa, que após atestamento ficou disponibilizado para o uso no país. O protocolo recomenda avaliar a presença/ausência de retalho da pele.

Ele é constituído de guia de tratamento, sistema de classificação e glossário. O guia de tratamento conta com seis tópicos relacionados aos cuidados com a ferida e a pele ao redor. O sistema de classificação avalia a presença/ausência do retalho de pele e sua viabilidade. Possui cinco fotografias, cada uma relacionada a sua respectiva descrição de categoria de Skin Tears. Por último, no verso do instrumento há o glossário, que traz as definições de Skin Tears e de termos técnicos relacionados ao assunto (Pinheiro et al., 2021).

No que se refere a busca pela padronização dos cuidados da LFs, estão surgindo outras iniciativas inovadoras, como por exemplo, a revisão de literatura realizada por Salomé et al., (2020), no qual resultou na formulação de uma cartilha validada, contendo em seus tópicos conceito, medidas preventivas e condutas terapêuticas, com o objetivo de auxiliar os profissionais no cuidado ao indivíduo que apresentam fatores de risco para a lesão, como também promover o processo de cicatrização das lesões já existentes.

Outro exemplo foi o algoritmo para a prevenção e tratamento das LFs formulado no estudo de Pinheiro et al., (2021) apontando os fatores de risco intrínseco e extrínseco para a ocorrência da lesão, a importância da reavaliação, além das condutas terapêuticas que vão desde a limpeza até a escolha das coberturas de acordo com a classificação da LFs. 
Neste cenário, o estabelecimento do POP para a sistematização da assistência no que tange às LFs estimula o trabalho multiprofissional durante atividades de cuidados preventivos e terapêuticos baseados em fatores de risco, vulnerabilidade e minimizando seus riscos e complicações. Os cuidados expressos no POP para atividades preventivas poderiam incluir a manutenção da homeostase orgânica e tecidual, focado na nutrição e hidratação, evitar traumas e proporcionar um ambiente seguro com dispositivos adequados. No que tange ao tratamento deve-se basear na classificação da lesão, por meio da utilização de instrumentos que permitam a escolha do curativo ideal.

Desse modo, faz-se relevante o uso de diretrizes, protocolos, manuais, fluxogramas e cartilhas para uma assistência à saúde de qualidade, através da padronização dos procedimentos clínicos e terapêuticos, como também o conhecimento teórico dos profissionais de saúde acerca da LFs mostra-se fundamental para uma correta e efetiva utilização desses protocolos, propiciando uma prática baseada em evidências e aumentando a probabilidade do sucesso na prevenção, manuseio e tratamento das LFs.

\section{Conclusão}

Por meio desta revisão foi possível caracterizar as produções científicas sobre as LFs ou do tipo Skin Tears. Verificouse que esse tipo de lesão apresenta alta prevalência, especialmente na população idosa, que as práticas de cuidados devem estar voltadas para a prevenção e manejo adequado a fim de evitar possíveis complicações e que a utilização de protocolos específicos que padronizem o cuidado das Skin Tears é fundamental para melhoria da qualidade da assistência.

A síntese das evidências encontradas colabora para o aprofundamento e disseminação de conhecimento sobre a temática em questão e para a construção de uma práxis de enfermagem baseada no saber científico. Como limitação da pesquisa, observouse uma escassez de estudos, principalmente de caráter experimental. Nesse sentido, recomenda-se a realização de futuros trabalhos para formação de mais pesquisas experimentais randomizadas buscando resultados mais robustos que subsidie uma assistência de enfermagem mais eficaz, mais segura, mais humanizada e com maior qualidade.

\section{Referências}

Amaral, A. F. dos S., Pulido, K. C. S., \& Santos, V. L. C. de G. (2012). Prevalência de lesões por fricção em pacientes hospitalizados com câncer. Revista Da Escola de Enfermagem Da USP, 46(spe), 44-50. https://doi.org/10.1590/s0080-62342012000700007

Aranha, J. D. S., Silva, J. L. L., Almeida, G. L., Lima, V. F., Santos, G. L. A., Souza, R. F., Oliveira, F. A. L. et al. (2020). Intervenções de enfermagem na prevenção de SkinTears. Brazilian Journal of Development, 6, 36849-36860. https://www.brazilianjournals.com/index.php/BRJD/article/view/11547/9715.

Carville, K., Lewin, G., Newall, N., Haslehurst, P., Michael, R., Santamaria, N., Roberts, P. (2007). STAR: a consensus for skin tear classification. Primary Intention, 15(1), 18-28. https://doi.org/20.500.11937/22957/117077-4723.

Faria, F. A. C. (2010). Escolas promotoras de saúde na América Latina: uma revisão integrativa da literatura. Dissertação (Mestrado em Promoção da Saúde) Universidade de Franca, Franca, SP. Brasil.

Fuchs, S. C., \& Paim, B. S. (2010). Meta-Analysis and systematic review of observational studies. Rev HCPA, 30(3), 294-301. https://www.researchgate.net/publication/279438697_Revisao_Sistematica_de_Estudos_Observacionais_com_Metanalise.

Geraldo Magela Salomé, José Carlos Bueno, \& Lydia Masako Ferreira. (2017). Aplicativo multimídia em plataforma móvel para tratamento de feridas utilizando fitoterápicos e plantas medicinais. Revista de Enfermagem UFPE on Line, 11(11), 4579-4588. https://doi.org/10.5205/1981-8963-v11i11a231197p4579-45882017

Gomes, B. E., Souza, P. V., Silva, G. D., Rocha, R. M., Kuriyama, S. N., \& Silvino, Z. R. (2016). Sistematizando o conhecimento acerca da prevenção das lesões do tipo Skin Tears na pele senil. Revista enfermagem atual in derme, 77, 75-81. http://www.revistaenfermagematual.com/index.ph p/revista/article/download/377/259 DOI: https://doi.org/10.31011/reaid-2016-v.77-n.15-art.377

Holmes, R. F., Davidson, M.W., Thompson, B. J., \& Kelechi, T. J. (2013). Skin Tears: Care and managemente of the Older adult at home. Home Healthcare Nurse, 31(2), 90-101. https://pubmed.ncbi.nlm.nih.gov/23385174/ DOI: 10.1097/NHH.0b013e31827f458a

Instituto Brasileiro de Geografia e Estatística - IBGE. (2017). Recuperado de https://agenciadenoticias.ibge.gov.br/agencia-noticias/2012-agencia-denoticias/noticias/20980-numero-de-idosos-cresce-18-em-5-anos-e-ultrapassa-30-milhoes-em-2017

Kimberly, K. C., \& Woo, L. K. (2018). Best practice recommendations for the prevention and management of Skin Tears in aged skin: an overview. Wounds International, 9, 66-70. https://www.woundsinternational.com/uploads/resources/5cd2a0928a6a935aef5389ce961fce44.pdf 
Leblanc, K., Baranoski, S., Christensen, D., Langemo, D., Sammon, M. A., Edwards, K., Holloway, S. et al. (2013). International Skin Tear Advisory Panel: A tool kit to aid in the prevention, assessment, and treatment of Skin Tears using a simplified classification system. Advances in Skin\&WoundCare, 26(10), 459476. https://pubmed.ncbi.nlm.nih.gov/24045566/ https://doi.org/10.1097/01.ASW. 0000434056.04071.68

Lima, M. I. V. de O., Pereira, J. de K. C., Soares, F. C., Farias, M. G. do N., Paz, E. B. R., Reis, A. M., Costa, E. B., Santos, M. L. M., \& Rocha, P. S. da S. (2021). Pressure injury in patients with advanced age and nursing care: An integrative literature review. Research, Society and Development, 10(5), e16310513373. https://doi.org/10.33448/rsd-v10i5.13373

Lopez, V., Dunk, A. M., Parke, J., Larkin, D., Trudinger, M., \& Stuart, M. (2011). Skin tear prevention and management among patients in the acute aged care and rehabilitation units in the Australian Capital Territory: a best practice implementation project. Int J Evid Based Healthc, 9(4), 429-434. https://pubmed.ncbi.nlm.nih.gov/22093391/ 10.1111/j.1744 1609.2011.00234.x.

Melnyk, B. M., Fineout-Overholt, E., Stillwell, S. B., \& Williamson, K. M. (2010). Evidence-based practice: step by step: The Seven Steps of Evidence-Based Practice. Am. j. Nurs, 110(1), 51-3. https://pubmed.ncbi.nlm.nih.gov/20032669/ https://doi.org/10.1097/01.NAJ.0000366056.06605.d2.

Payne R. L.; Martin, M. L. (2017). The epidemiology and management of skin tears in older adults. Ostomy/Wound Management, 26. https://doi.org/10.1097/01.NAJ.0000505582.01967

Pinheiro, R. V., Salomé, G. M., Miranda, F. D., Alves, J. R., Reis, F. A. dos, \& Mendonça, A. R. dos A. (2021). Algoritmos para prevenção e tratamento de lesão por fricção. Acta Paulista de Enfermagem, 34. https://doi.org/10.37689/acta-ape/2021ao03012

Powell, R. J., Hayward, C. J., Snelgrove, C. L., Polverino, K., Park, L., Chauhan, R., Evans, P. H., Byford, R., Charman, C., Foy, C. J. W., \& Kingsley, A. (2015). Pilot randomised controlled trial of protective socks against usual care to reduce skin tears in high risk people "STOPCUTS": study protocol. Pilot and Feasibility Studies, 1(1). https://doi.org/10.1186/s40814-015-0005-3

Soh, Z., Wang, W., Kheng, G., Hassan, N., \& Wu, V. X. (2019). Risk of Skin Tears and its predictors among hospitalized older adults in Singapore. Int J NursPract, 25, 1-11. https://pubmed.ncbi.nlm.nih.gov/31793134/DOI: 10.1111 / ijn.12790.

Torres, F. S., Blanes, L., Galvão, T. F., \& Ferreira, L. M. (2017). Manual de prevenção e tratamento de lesões por fricçã. [Manual]. São Paulo, SP. https://www.portaldaenfermagem.com.br/downloads/MANUAL_LESOES_POR_FRICCAO_2017_comISBN.pdf

Tristão, F. R., Girondi, J. B. R., Hammerschmidt, K. S. A., Soares, C. F., Martins, T., \& Lima, D. K. S. (2018). Risco para LFs em idosos longevos na atenção primária à saúde. ESTIMA, Braz. J. Enterostomal Ther, 16, 1-7. Recuperado de https://www.revistaestima.com.br https://doi.org/10.30886/estima.v16.614_PT.

Soares, C. B., Hoga, L. A. K., Peduzzi, M., Sangaleti, C., Yonekura, T., \& Silva, D. R. A. D. (2014). Revisão integrativa: conceitos e métodos utilizados na enfermagem. Rev esc. enferm. USP, 48(2), 335-45. https://www.scielo.br/j/reeusp/a/3ZZqKB9pVhmMtCnsvVW5Zhc/?lang=pt https://doi.org/10.1590/S00806234201400002000020

Strazieri-Pulido K. C. Adaptação cultural e validação do instrumento "Star Skin Tear Classification System”, para a língua portuguesa no Brasil. Dissertação]. Escola de Enfermagem, Universidade de São Paulo; 2010 